\title{
Nesfatin-1 suppresses peripheral arterial remodeling without elevating blood pressure in mice
}

\author{
Yusaku Mori ${ }^{1, *}$, Hiroyuki Shimizu ${ }^{1,2, *}$, Hideki Kushima ${ }^{1}$, Tomomi Saito ${ }^{1}$, Munenori Hiromura ${ }^{1}$, Michishige Terasaki ${ }^{1}$, \\ Masakazu Koshibu' ${ }^{1}$ Hirokazu Ohtaki ${ }^{3}$ and Tsutomu Hirano' ${ }^{1}$ \\ ${ }^{1}$ Division of Diabetes, Metabolism, and Endocrinology, Department of Internal Medicine, Showa University School of Medicine, Shinagawa, Tokyo, Japan \\ ${ }^{2}$ Maebashi Hirosegawa Clinic, Maebashi, Gunma, Japan \\ ${ }^{3}$ Department of Anatomy, Showa University School of Medicine, Shinagawa, Tokyo, Japan
}

Correspondence should be addressed to Y Mori: u-mori@med.showa-u.ac.jp

*(Y Mori and $\mathrm{H}$ Shimizu contributed equally to this work)

\begin{abstract}
Nesfatin-1 is a novel anorexic peptide hormone that also exerts cardiovascular protective effects in rodent models. However, nesfatin-1 treatment at high doses also exerts vasopressor effects, which potentially limits its therapeutic application. Here, we evaluated the vasoprotective and vasopressor effects of nesfatin-1 at different doses in mouse models. Wild-type mice and those with the transgene nucleobindin-2, a precursor of nesfatin-1, were employed. Wild-type mice were randomly assigned to treatment with vehicle or nesfatin-1 at $0.2,2.0$ or $10 \mu \mathrm{g} / \mathrm{kg} /$ day (Nes-0.2, Nes-2, Nes-10, respectively). Subsequently, mice underwent femoral artery wire injury to induce arterial remodeling. After 4 weeks, injured arteries were collected for morphometric analysis. Compared with vehicle, nesfatin-1 treatments at 2.0 and $10 \mu \mathrm{g} / \mathrm{kg} /$ day decreased body weights and elevated plasma nesfatin-1 levels with no changes in systolic blood pressure. Furthermore, these treatments reduced neointimal hyperplasia without inducing undesirable remodeling in injured arteries. However, nesfatin-1 treatment at $0.2 \mu \mathrm{g} / \mathrm{kg} /$ day was insufficient to elevate plasma nesfatin-1 levels and showed no vascular effects. In nucleobindin-2transgenic mice, blood pressure was slightly higher but neointimal area was lower than those observed in littermate controls. In cultured human vascular endothelial cells, nesfatin-1 concentration-dependently increased nitric oxide production. Additionally, nesfatin-1 increased AMP-activated protein kinase phosphorylation, which was abolished by inhibiting liver kinase B1. We thus demonstrated that nesfatin-1 treatment at appropriate doses suppressed arterial remodeling without affecting blood pressure. Our findings indicate that nesfatin-1 can be a therapeutic target for improved treatment of peripheral artery disease.
\end{abstract}

\section{Key Words}

- AMP-activated protein kinase

- arterial remodeling

- blood pressure

- neointima

- nesfatin-1

\section{Introduction}

Nesfatin-1 is a novel peptide hormone that suppresses appetite through its actions in the central nervous system $(1,2)$. Additionally, nesfatin-1 is directly involved in the regulation of the cardiovascular system $(2,3)$.
Cohort studies, albeit not all, have shown that lower levels of nesfatin-1 in the circulation are associated with cardiovascular disease (CVD) and risk factors for CVD including type 2 diabetes $(4,5,6,7,8)$. CVD is the

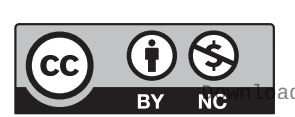

This work is licensed under a Creative Commons Attribution-NonCommercial 4.0 International License. ded from Bioscientifica.com at 04/26/2023 12:36:47PM 
main cause of death, especially in obesity-related diseases such as type 2 diabetes and metabolic syndrome, in many countries. For future application of nesfatin-1, it is important to clarify whether pharmacological concentrations of nesfatin- 1 can exert beneficial effects against CVD.

Preclinical studies have demonstrated cardiovascular protective and anti-inflammatory effects of nesfatin-1 treatment in rodent models $(9,10,11,12,13,14$, 15). These findings indicate that nesfatin- 1 may be a potential therapeutic target for CVD. Conversely, it is also reported that unfavorable cardiovascular changes such as hypertension are induced by nesfatin- 1 treatment at much higher doses than those exerting cardiovascular protective effects $(16,17,18)$, which potentially limits clinical application of nesfatin-1. However, it is unclear whether appropriate doses of nesfatin-1 can exert cardiovascular protective effects without inducing unfavorable cardiovascular changes.

In the present study, we aimed to evaluate the vasoprotective and vasopressor effects of nesfatin-1 at different doses in mouse models of peripheral arterial remodeling. Additionally, we sought to clarify the underlying mechanisms in cultured human vascular endothelial cells (VECs) and vascular smooth muscle cells (VSMCs).

\section{Materials and methods}

\section{Chemical agents}

Human nesfatin-1 was purchased from Sigma-Aldrich Japan (Product ID: SRP3291; Tokyo, Japan). Plateletderived growth factor (PDGF)-BB was obtained from Wako (Osaka, Japan).

\section{Animal studies}

The study design was approved by the Animal Care Committee of Showa University School of Medicine (approval numbers: 05031 and 07006). Animal experiments were conducted under strict adherence to the Guide for the Care and Use of Laboratory Animals (19). All invasive procedures were performed under general anesthesia using isoflurane. Male C57BL/6J (wildtype) mice were purchased from Sankyo Labo Service (Tokyo, Japan). Mice with the transgene nucleobindin-2, a precursor of nesfatin-1, were generated and backcrossed to the C57BL/6 strain as previously described (NUCB2-Tg mice) (20). Mice were maintained on standard rodent chow (Labo MR Stock, NOSAN, Yokohama, Japan) with free access to water and housed within a specific pathogenfree facility in the Division of Animal Experimentation of Showa University School of Medicine. The rooms were controlled under a 12 -h dark/light cycle, $21^{\circ} \mathrm{C}$ and $40-60 \%$ humidity. At 9 to 12 weeks of age (day 1), wild-type mice were randomly divided into treatment groups as follows: vehicle or nesfatin- 1 at a low dose $(0.2 \mu \mathrm{g} / \mathrm{kg} /$ day, Nes-0.2), moderate dose $(2.0 \mu \mathrm{g} / \mathrm{kg} /$ day, Nes-2), or high dose $(10 \mu \mathrm{g} / \mathrm{kg} /$ day, Nes-10). The doses of nesfatin-1 were determined based on previous studies reporting tissueprotective effects $(9,11,13,14,15)$. Vehicle or nesfatin-1 was delivered using osmotic pumps (Alzet minipump 1002; Cupertino, CA, USA), which were replaced every 14 days to avoid agent degradation. On day 3 , mice were subjected to femoral artery wire injury as previously described by Sata et al. (21), with a few modifications (22). Briefly, a straight spring wire was inserted in a retrograde manner into the femoral artery via a small cut on the muscle branch and withdrawn after 1 min. The muscle branch was ligated proximal to the cut to prevent bleeding, and the skin incision was sutured. Following the procedure, mice recovered in a separate cage for $6 \mathrm{~h}$ under careful observation, after which they were returned to their original cages. Experiments employing male NUCB2-Tg mice and littermate controls were similarly performed without osmotic pump implantation. No mice showed signs of severe complications such as claudication or necrosis of the injured extremity, surgical site infection, or massive weight loss (20\% of baseline). Arterial remodeling in injured femoral arteries reaches its peak at 28 days after wire insertion in the present model (21). Mice were killed by an overdose of inhaled isoflurane on day 29, and blood and vessel samples were collected for analysis.

\section{Assessment of plasma levels}

Blood samples were collected after $6 \mathrm{~h}$ of fasting at the end of each experiment. Plasma glucose levels were measured using GlutestSensor Neo Super (Sanwa Kagaku Kenkyusho, Nagoya, Japan). Plasma lipid levels were determined using an enzymatic colorimetric assay (Cobas, Roche Diagnostics Japan). Plasma levels of nesfatin-1 and pentraxin 3 were measured by enzymelinked immunosorbent assay (ab213812, Abcam Japan; ARG81768, Arigo Biolaboratories, Hsinchu City, Taiwan).

\section{Measurement of blood pressure}

Systolic blood pressure and pulse rates were measured using the tail-cuff method (Model MK-2000ST; Muromachi

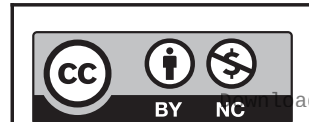

This work is licensed under a Creative Commons Attribution-NonCommercial 4.0 International License. ded from Bioscientifica.com at 04/26/2023 12:36:47PM via free access 
Kikai, Tokyo, Japan) (22). The average value obtained from three to five consecutive measurements was used as a single data point.

\section{Morphometric analysis}

Collected femoral arteries were embedded into paraffin blocks for morphometric analysis. To reduce selection bias, serial cross-sections were taken from the proximal end of each femoral artery at $0.5 \mathrm{~mm}$ intervals, and their average was used as a single data point. Sections stained with Elastica van Gieson (EVG) were analyzed using ImageJ software (National Institutes of Health) by an investigator who was blinded to the treatment. The areas within the internal elastic lamina and between the internal and external elastic lamina were defined as the neointima and media, and the perimeter of the external elastic lamina was defined as the artery perimeter (22). Sections with the following were excluded from the analysis: complete occlusion by thrombus, broken wall structure, a branch of another artery or a missing elastic lamina. There was no difference in the percentage of excluded sections between the groups (wild-type mice: vehicle, $27 \pm 8 \%$; Nes-0.2, $25 \pm 10 \%$; Nes-2, $28 \pm 8 \%$; Nes- 10 , $33 \pm 11 \%, P=0.95$ by one-way ANOVA; NUCB2-Tg and littermate control mice: control, $20 \pm 12 \%$; NUCB2-Tg, $25 \pm 13 \%, P=0.80$ by unpaired $t$-test).

\section{Immunohistochemistry}

Proliferating cells were identified by immunostaining using an anti-Ki67 antibody (Thermo Fisher Scientific Japan, RM-9106-S1, RRID: AB_149792, raised in rabbit, 1:250) (22). Nuclei were counterstained with hematoxylin. The average of four serial cross-sections was presented as a single data point.

\section{Cell culture}

Human umbilical vein endothelial cells (HUVECs; umbilical veins from pooled donors; authentication: positive for CD31, CD105, Axl, endothelial nitric oxide synthase, Tie-2, and vascular endothelial growth factor receptor 2) and human aortic smooth muscle cells (HASMCs; authentication: positive for smooth muscle $\alpha$-actin and negative for von Willebrand antigen) were obtained from Lonza Japan (Tokyo, Japan) and cultured in EGM-2 (PromoCell GmbH, Sickingenstr, Heidelberg, Germany) and SmBM (Lonza Japan), respectively. For protein extraction, cells were seeded onto 12-well plates at a density of $1 \times 10^{5}$ cells per well. After $24 \mathrm{~h}$, cells were serum-starved in M199 medium (Thermo Fisher Scientific Japan) containing $0.5 \%$ bovine serum albumin (BSA) for $6 \mathrm{~h}$ and stimulated with $100 \mathrm{nmol} / \mathrm{L}$ nesfatin- 1 for the indicated time periods $(18,23,24,25)$.

\section{Measurement of nitric oxide (NO) production}

HUVECs were seeded onto 24-well plates at a density of $2.5 \times 10^{4}$ cells per well. After $24 \mathrm{~h}$, cells were serum-starved for $1 \mathrm{~h}$ in M199 medium containing 0.3\% fetal bovine serum (FBS) and subsequently incubated with the indicated concentrations of nesfatin- 1 for $2 \mathrm{~h}$. NO production was determined by evaluating the stable metabolites of $\mathrm{NO}$ $\left(\mathrm{NO}_{2}\right.$ and $\left.\mathrm{NO}_{3}\right)$ in the culture medium (Nitrate/Nitrite Fluorometric Assay Kit; Dojindo, Kumamoto, Japan) as previously described (22). The fluorescence intensities were measured using an Infinite M200 PRO instrument (TECAN Japan, Kawasaki, Japan).

\section{Cell proliferation}

HUVECs and HASMCs were seeded onto 24-well plates at densities of $2.5 \times 10^{4}$ cells and $1 \times 10^{4}$ cells per well, respectively (26). HUVECs were serum-starved in M199 medium containing $0.3 \%$ FBS for $4 \mathrm{~h}$ and incubated with the indicated concentrations of nesfatin-1 for $24 \mathrm{~h}$. HASMCs were serum-starved overnight in SmBM (growth factor- and serum-free) containing 0.3\% BSA and incubated with the indicated concentrations of nesfatin-1 in the absence or presence of PDGF-BB $(20 \mathrm{ng} / \mathrm{mL})$ for various time periods. WST- 8 reagent (Wako) was added to medium at a ratio of $1: 10$, and the absorbance at $450 \mathrm{~mm}$ was measured after a 2-h incubation using an Infinite M200 PRO instrument (TECAN Japan).

\section{Western blotting}

Protein levels were determined by western blotting as previously described (27). Briefly, $4.5 \mu \mathrm{g}$ of extracted protein was electrophoresed in polyacrylamide gels and transferred to PVDF membranes. After blocking with PVDF-blocking reagent (TOYOBO, Osaka, Japan) for $1 \mathrm{~h}$, the membranes were incubated with primary antibodies overnight, followed by incubation with secondary antibody for $1 \mathrm{~h}$. Antibody information is presented in Supplementary Table 1 (see section on supplementary data given at the end of this article). The bands on the immunoblot were detected using the Amersham ECL Prime kit (GE Healthcare Japan), digitized using WSE-6100

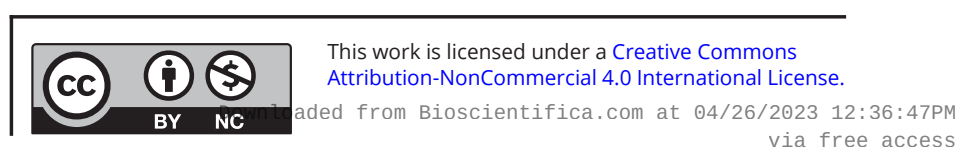


LuminoGraph (ATTO, Tokyo, Japan), and quantified using CS Analyzer 4 software (ATTO).

\section{siRNA transfection}

Predesigned siRNAs for the negative control (Silencer Select Negative Control No. 2 siRNA) and targeting liver kinase B1 (LKB1) were purchased from Thermo Fisher Scientific Japan (Silencer Select Negative Control No. 2 siRNA; LKB1 siRNA S74498). A mixture of siRNA and transfection reagent (TransIT-TKO; Mirus Bio, Madison, WI, USA) was added to medium at a final siRNA concentration of $12.5 \mathrm{nmol} / \mathrm{L}$ (27). Cells were used for experiments $72 \mathrm{~h}$ after siRNA transfection.

\section{Statistical analysis}

Data are expressed as means \pm S.E.M. Comparisons were conducted using an unpaired $t$-test for two groups and oneway or two-way ANOVA followed by Tukey's test for three or more groups. The Pearson correlation coefficient was used to test correlations between variables. Statistical calculations were performed using JMP software (version 13; SAS Institute Inc.). The significance level was defined as $P<0.05$.

\section{Results}

\section{Nesfatin-1 treatment at doses exerting no vasopressor effect suppresses peripheral arterial remodeling}

First, we evaluated the effects of nesfatin-1 on peripheral arterial remodeling in wild-type mice. Both Nes-2 $(2 \mu \mathrm{g} / \mathrm{kg} /$ day $)$ and Nes-10 (10 $\mu \mathrm{g} / \mathrm{kg} /$ day $)$ treatment decreased final body weights and elevated plasma nesfatin-1 levels compared with vehicle treatment (Fig. 1A and Table 1). Nes-10 treatment also increased water intake, which was consistent with our previous findings in NUCB-2 Tg mice (Table 1) (28). However, other parameters including pulse rate and systolic blood pressure were not affected (Fig. 1B, C and Table 1). Additionally, plasma levels of pentraxin 3, a marker of systemic inflammation, were similar between groups (Table 1). Conversely, in the Nes-0.2 group $(0.2 \mu \mathrm{g} / \mathrm{kg} /$ day $)$, plasma nesfatin- 1 levels were not elevated (Fig. 1A), and there was no difference in physiological or biochemical parameters (Fig. 1A, B, C and Table 1).

Analysis of histological changes revealed that both Nes-2 and Nes-10 treatment similarly suppressed neointimal hyperplasia without inducing undesirable remodeling such as medial thinning or arterial dilation. Additionally, Nes-10 treatment reduced the ratio of intimal area to medial area (I/M ratio), and Nes-2 treatment showed a trend toward a decrease in this ratio $(P=0.05$, Fig. $1 \mathrm{D}, \mathrm{E}, \mathrm{F}, \mathrm{G}$ and $\mathrm{H})$. In contrast, Nes-0.2 treatment did not show any effect on vascular parameters.

\section{Overexpression of NUCB2 leads to the suppression of neointimal hyperplasia}

Next, we evaluated peripheral arterial remodeling in mice overexpressing NUCB2, a precursor of nesfatin-1. In NUCB2-Tg mice, blood pressure was slightly elevated as previously reported (28); however, other physiological and metabolic parameters were similar to those in littermate controls (Table 2). In injured arteries, neointimal area was significantly reduced in NUCB2-Tg mice compared with that observed in littermate controls (Fig. 2A and $\mathrm{B})$, whereas the medial area and arterial perimeter were similar between controls and NUCB2-Tg mice (Fig. 2C and $\mathrm{D}$ ). These changes resulted in a reduced $\mathrm{I} / \mathrm{M}$ ratio in NUCB2-Tg mice (Fig. 2E).

Subsequently, we assessed vascular cell proliferation, which plays a crucial role in the process of neointimal hyperplasia (29). In NUCB2-Tg mice, the percentages of Ki-67-positive cells both in the neointima and media were significantly reduced compared with those in littermate controls (Fig. 2F, G and H). Furthermore, there was a significant correlation between the number of Ki-67positive cells and neointimal area $(r=0.463, P=0.029)$. Conversely, neointimal and medial cell densities, which were calculated as area per cell, were similar between controls and NUCB2-Tg mice (Fig. 2I and J).

\section{Nesfatin-1 increases NO production and AMPK phosphorylation in HUVECs}

Following the animal experiments, we performed in vitro experiments. First, we evaluated effects of nesfatin-1 on VECs. Nesfatin-1 at concentrations of $0.1-10 \mathrm{nmol} / \mathrm{L}$ dose-dependently increased NO production (Fig. 3A). However, nesfatin-1 did not affect cell proliferation (Fig. 3B)

Subsequently, we investigated molecules potentially involved in the NO-producing effects of nesfatin-1. Nesfatin-1 significantly increased phosphorylated AMPK levels (phosphorylated AMPK/total AMPK (p-AMPK/t-AMPK), Fig. 3D; $\quad$-AMPK/ $\beta$-actin, Supplementary Fig. 1A), whereas neither phosphorylated Akt nor p42/44 levels were changed (p-Akt/t-Akt 

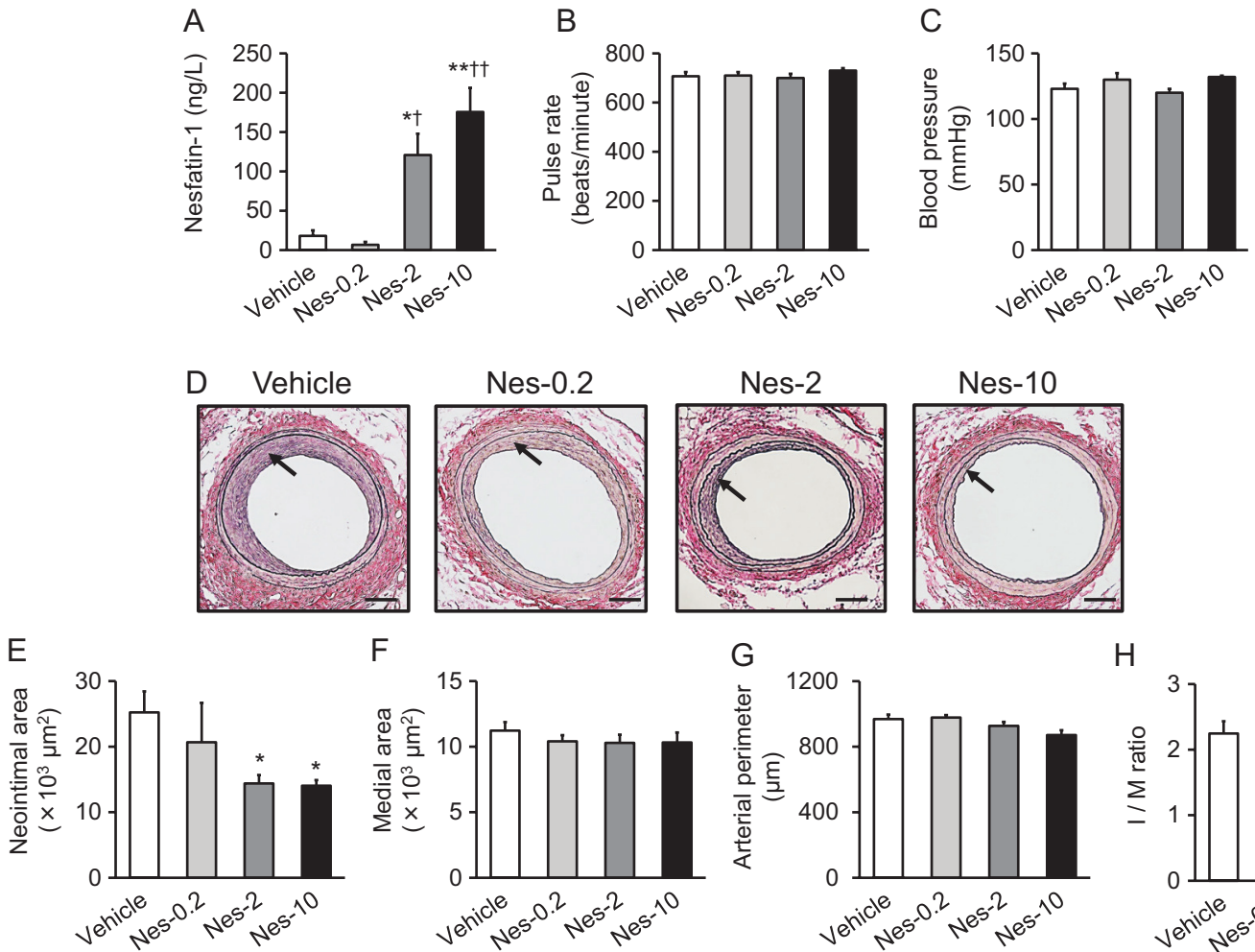

F
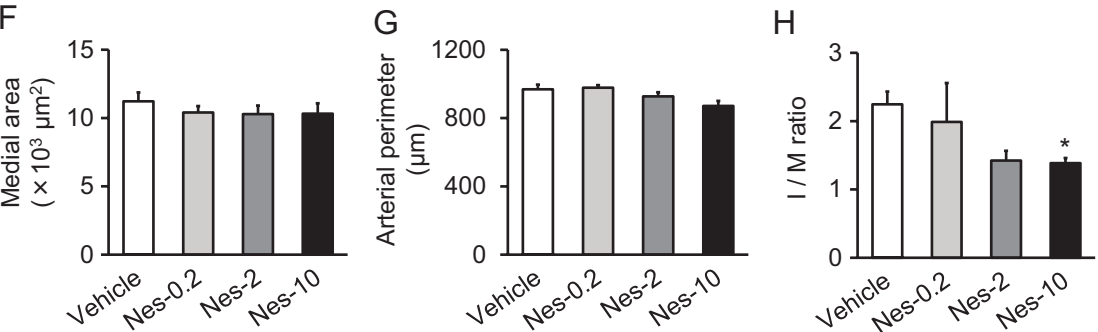

Figure 1

Nesfatin-1 treatment suppresses arterial remodeling without affecting blood pressure in wild-type mice. Wild-type mice were treated with vehicle or

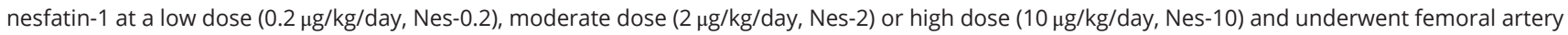
wire injury. (A) Plasma levels of nesfatin-1. (B) Pulse rate. (C) Systolic blood pressure. (D) Representative images of EVG-stained femoral arteries. Scale bars $=100 \mu \mathrm{m}$. Arrows indicate neointima. (E) Neointimal area. (F) Medial area. (G) Arterial perimeter. (H) I/M ratio. Vehicle, $n=7 ; \mathrm{Nes}-0.2, n=4 ;$ Nes-2, $n=7$; Nes-10, $n=7 .{ }^{*} P<0.05$ vs vehicle; ${ }^{* *} P<0.01$ vs vehicle; ${ }^{\dagger} P<0.05$ vs Nes-0.2; ${ }^{+t} P<0.01$ vs Nes-0.2.

and p-p42/44/t-p42/44, Fig. 3E and F; p-Akt/ $\beta$-actin, Supplementary Fig. 1 C; p-p42/ $\beta$-actin and p-p44/ $\beta$-actin, Supplementary Fig. 1E and F). Total protein levels of AMPK, Akt, p42 and p44 were not changed by nesfatin-1 stimulation (Supplementary Fig. 1B, D, G and H). To obtain a clearer understanding of the underlying mechanism, we evaluated the involvement of LKB1, a molecule that acts upstream of AMPK phosphorylation. Upon siRNA-mediated knockdown of LKB1 protein levels by over 95\% (Fig. 3G), nesfatin-1 failed to increase AMPK phosphorylation (Fig. 3G and H).

\section{Nesfatin-1 increases AMPK phosphorylation but not cell proliferation in HASMCs}

Next, we assessed the effects of nesfatin-1 on VSMCs. HASMCs were stimulated with nesfatin-1 at different concentrations in the presence of PDGF for $48 \mathrm{~h}$; however, nesfatin-1 did not affect PDGF-induced cell proliferation (Fig. 4A). Furthermore, in the assessment of time-dependent effects, neither 100 nor $1000 \mathrm{nmol} / \mathrm{L}$ nesfatin-1 affected cell proliferation at any time point, regardless of stimulation with PDGF (Fig. 4B). Conversely, nesfatin-1 significantly increased phosphorylated AMPK levels in HASMCs, as was observed in HUVECs (p-AMPK/t-AMPK, Fig. $\quad 4 \mathrm{D} ; \quad$-AMPK/ $\beta$-actin, Supplementary Fig. 1I), whereas phosphorylated Akt and p42/44 levels were not changed (p-Akt/t-Akt and p-p42/44/t-p42/44, Fig. $4 \mathrm{E}$ and F; p-Akt/ $\beta$-actin, Supplementary Fig. $1 \mathrm{~K}$; p-p42/ $\beta$-actin and p-p44/ $\beta$-actin, Supplementary Fig. $1 \mathrm{M}$ and $\mathrm{N}$ ). Total protein levels of AMPK, Akt, p42 and p44 were not changed by nesfatin-1 stimulation (Supplementary Fig. 1J, L, O and P).

\section{Discussion}

In the present study, we demonstrated that nesfatin-1 treatment at doses of 2 and $10 \mu \mathrm{g} / \mathrm{kg} /$ day increased plasma nesfatin-1 levels and suppressed peripheral arterial remodeling without elevating systolic blood pressure in mouse models of femoral artery wire injury.

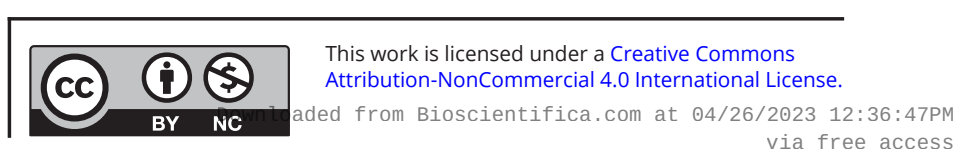


Table 1 Physiological and biochemical parameters of mice treated with vehicle or nesfatin-1.

\begin{tabular}{l}
\hline Number \\
Food intake (g/day) \\
Fluid intake (ml/day) \\
Initial body weight (g) \\
Final body weight (g) \\
Liver/BW (mg/g) \\
Epididymal fat/BW (mg/g) \\
Glucose level (mg/dL) \\
Total cholesterol level (mg/dL) \\
HDL-cholesterol level (mg/dL) \\
Triglyceride level (mg/dL) \\
Pentraxin 3 level (pg/mL) \\
\hline
\end{tabular}

\begin{tabular}{c}
\hline Vehicle \\
\hline 7 \\
$3.9 \pm 0.1$ \\
$4.9 \pm 0.1$ \\
$25.6 \pm 0.8$ \\
$26.4 \pm 0.7$ \\
$46.9 \pm 2$ \\
$12.5 \pm 0.9$ \\
$114 \pm 6$ \\
$84 \pm 5$ \\
$68 \pm 5$ \\
$56 \pm 4$ \\
$2790 \pm 401$ \\
\hline
\end{tabular}

\begin{tabular}{c}
\hline Nes-0.2 \\
\hline 4 \\
$3.8 \pm 0.2$ \\
$5.1 \pm 0.3$ \\
$25.4 \pm 0.6$ \\
$24.8 \pm 0.3$ \\
$46.2 \pm 4.1$ \\
$10.2 \pm 0.8$ \\
$116 \pm 15$ \\
$81 \pm 5$ \\
$65 \pm 6$ \\
$54 \pm 2$ \\
$2189 \pm 124$ \\
\hline
\end{tabular}

\begin{tabular}{c}
\hline Nes-2 \\
\hline 7 \\
$3.6 \pm 0.2$ \\
$4.9 \pm 0.3$ \\
$24.0 \pm 0.5$ \\
$23.6 \pm 0.4^{b}$ \\
$43.9 \pm 1.3$ \\
$11.2 \pm 1.0$ \\
$101 \pm 5$ \\
$85 \pm 5$ \\
$69 \pm 6$ \\
$55 \pm 2$ \\
$2245 \pm 94$ \\
\hline
\end{tabular}

\begin{tabular}{c}
\hline Nes-10 \\
\hline 7 \\
$4.4 \pm 0.1$ \\
$6.3 \pm 0.1^{\mathrm{a}}$ \\
$23.2 \pm 0.9$ \\
$24.0 \pm 0.7^{\mathrm{b}}$ \\
$45.4 \pm 1.5$ \\
$9.5 \pm 0.5$ \\
$100 \pm 9$ \\
$84 \pm 4$ \\
$67 \pm 4$ \\
$54 \pm 2$ \\
$2191 \pm 151$
\end{tabular}

Values are shown as mean \pm S.E.M.

a $P<0.05$ vs all; ${ }^{b} P<0.05$ vs vehicle.

BW, body weight; HDL, high-density lipoprotein; Nes-0.2, nesfatin-1 at $0.2 \mu \mathrm{g} / \mathrm{kg} / \mathrm{day} ; \mathrm{Nes}-2$, nesfatin-1 at $2 \mu \mathrm{g} / \mathrm{kg} / \mathrm{day} ; \mathrm{Nes-10,} \mathrm{nesfatin-1} \mathrm{at} 10 \mu \mathrm{g} / \mathrm{kg} / \mathrm{day}$.

Of note, treatment at $10 \mu \mathrm{g} / \mathrm{kg} / \mathrm{day}$ did not lead to a greater suppression in arterial remodeling than that at $2 \mu \mathrm{g} / \mathrm{kg} /$ day, whereas plasma nesfatin-1 levels differed. Thus, it is possible that the maximal vasoprotective effects of nesfatin- 1 were obtained by treatment at $2 \mu \mathrm{g} / \mathrm{kg} /$ day. However, the vasoprotective and vasopressor effects of nesfatin- 1 at doses higher than $10 \mu \mathrm{g} / \mathrm{kg} /$ day need to be determined in a future study.

In previous studies reporting the vasopressor effects of nesfatin-1, nesfatin-1 was administered to rodents by intraperitoneal injection of more than $250 \mathrm{nmol} / \mathrm{kg}$, which is a much higher dose than those used in the present study (the highest dose of $10 \mu \mathrm{g} / \mathrm{kg} /$ day is equal to approximately $1 \mathrm{nmol} / \mathrm{kg} /$ day) $(16,17,18)$. However, plasma nesfatin-1 levels in rodents, as measured by ELISA, vary widely among studies, possibly due to non-specific detection. Thus, plasma nesfatin-1 levels that exert vasopressor effects have not been determined. Compared with rodents, plasma nesfatin-1 levels in humans are relatively consistent between studies; differences in plasma/serum nesfatin-1 levels between individuals with and without targeted diseases are on the order of approximately $200 \mathrm{ng} / \mathrm{L}(4,5,8)$. It has been shown that human nesfatin-1 exerts similar effects to mouse nesfatin- 1 in rodents (30). Thus, in the present study, we administered human nesfatin-1 to mice and measured plasma nesfatin-1 levels with a commercially available ELISA kit for human nesfatin-1. We found that plasma nesfatin-1 levels were increased in tandem with escalating doses of human nesfatin-1 administration. However, the cross-reactivity to mouse nesafatin-1 in this ELISA kit has not been not determined, and plasma nesfatin-1 levels in vehicle-treated mice might not exactly represent baseline levels. Therefore, instead of absolute values, the net increases in plasma nesfatin-1 levels can be compared: 100 and $150 \mathrm{ng} / \mathrm{L}$ following nesfatin- 1 treatment at 2 and $10 \mu \mathrm{g} / \mathrm{kg} /$ day, respectively. Thus, it is plausible to postulate that the observed increases in plasma nesfatin-1 levels were clinically relevant changes and could lead to vasoprotection without inducing hypertension.

In NUCB2-Tg mice, arterial remodeling was suppressed compared with that in littermate controls. Furthermore, this effect was associated with decreased neointimal cell proliferation, whereas the neointimal cell density was similar. However, we did not find direct anti-mitotic effects of nesfatin-1 in HASMCs regardless of stimulation with PGDF. These findings indicate that the suppression of vascular cell proliferation observed in vivo was likely to be induced by indirect mechanisms.

Using cultured HUVECs, we found that nesfatin-1 directly increased the production of NO, which has been shown to inhibit vascular remodeling including neointimal

Table 2 Physiological and biochemical parameters of NUCB2-Tg mice and littermate controls.

\begin{tabular}{|c|c|c|}
\hline & Control & NUCB2-Tg \\
\hline Number & 6 & 7 \\
\hline Food intake (g/day) & $4.2 \pm 0.2$ & $4.8 \pm 0.3$ \\
\hline Fluid intake (mL/day) & $5.1 \pm 0.3$ & $5.2 \pm 0.3$ \\
\hline Initial body weight (g) & $27.5 \pm 0.3$ & $27.5 \pm 0.5$ \\
\hline Final body weight (g) & $27.7 \pm 0.7$ & $27.2 \pm 0.6$ \\
\hline Weight increase (g) & $0.0 \pm 0.5$ & $-0.3 \pm 0.2$ \\
\hline Pulse rate (beats/min) & $696 \pm 13$ & $701 \pm 12$ \\
\hline $\mathrm{SBP}(\mathrm{mmHg})$ & $104 \pm 3$ & $127 \pm 9^{a}$ \\
\hline Glucose level (mg/dL) & $130 \pm 8$ & $112 \pm 7$ \\
\hline Pentraxin 3 level (pg/mL) & $1522 \pm 52$ & $1507 \pm 157$ \\
\hline
\end{tabular}

Values are shown as mean \pm S.E.M.

a $P<0.05$ vs control.

NUCB2-Tg, nucleobindin-2 transgenic; SBP, systolic blood pressure.

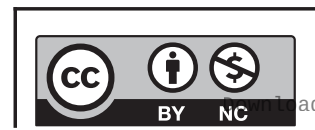

This work is licensed under a Creative Commons Attribution-NonCommercial 4.0 International License. ded from Bioscientifica com at 04/26/2023 12:36:47PM 
A
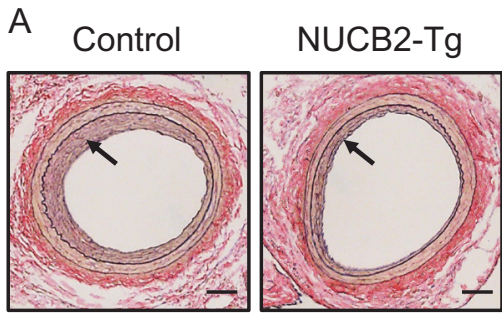

$B$

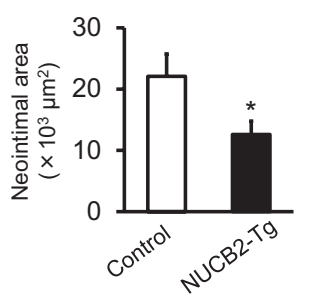

E
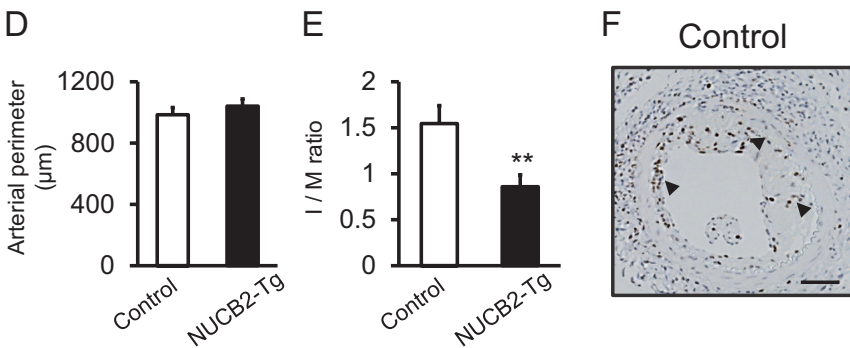

।
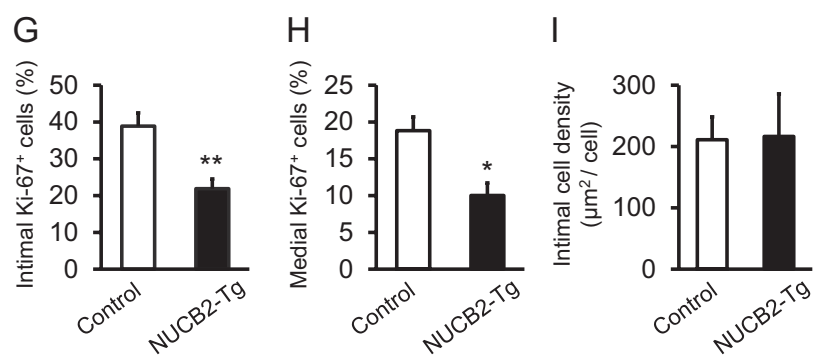

hyperplasia via the suppression of VSMC proliferation (31, $32,33,34$ ). Barutcigil et al. (23) have demonstrated that nesfatin-1 dose-dependently induces relaxation responses in isolated rat aortas. In this study, the effects of nesfatin-1 were attenuated by the removal of endothelium and the inhibition of NO synthase or soluble guanylate cyclase, indicating endothelial NO-dependent mechanisms. In our model, VECs are removed from the lumen immediately after wire insertion (27). However, VECs outside of the wireinserted segment remain intact, unlike in other models of vascular remodeling induced by systemic changes. In addition, we previously reported that removed VECs were rapidly regenerated and covered the lumen prior to the formation of the neointima (27). Taken together with the findings that nesfatin-1 did not show direct anti-mitotic effects in HASMCs, the enhancement of NO production from VECs in intact arteries and regenerated VECs in injured arteries is likely to be involved in the anti-arterial remodeling effects of nesfatin-1.

Zhang et al. (18) reported that adenovirus-mediated knockdown of nesfatin-1 led to the suppression of arterial remodeling in a rat model of artery ligation. In this model, blood flow in the common carotid artery was completely disrupted by ligation near the bifurcation.
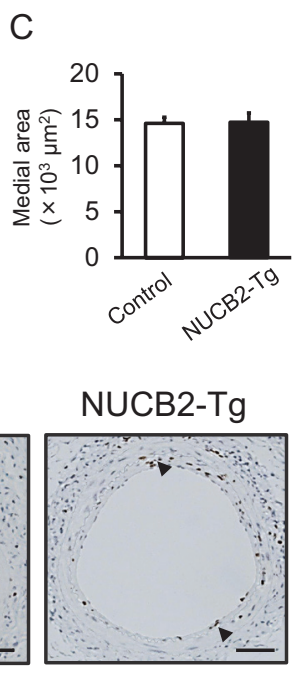

Figure 2

Overexpression of NUCB2 leads to suppression of neointimal hyperplasia. NUCB2-Tg mice and littermate controls underwent femoral artery wire injury. (A) Representative images of EVG-stained femoral arteries. Scale bars $=100 \mu \mathrm{m}$. Arrows indicate neointima. (B) Neointimal area. (C) Medial area. (D) Arterial perimeter. (E) I/M ratio. (A, B, C, $\mathrm{D}$ and E) Control, $n=6$; NUCB2-Tg, $n=7$. (F) Representative images of Ki-67-immunostained femoral arteries. Bars $=100 \mu \mathrm{m}$. Arrowheads indicate Ki-67-positive cells. ( $\mathrm{G}$ and $\mathrm{H}$ ) Percentage of Ki-67-positive cells to total cells in the neointima ( $\mathrm{G}$ ) and media (H). (I and J) Cell density in the neointima (I) and media (J). $n=4$ per group. $\star P<0.05,{ }^{*} P<<0.01$.

However, the ligated artery showed pulsation due to being subjected to arterial blood pressure, resulting in higher pressure on the vessel wall than under un-ligated conditions (35). It was also reported by the same group that adenovirus-mediated knockdown of nesfatin-1 prevented aortic dilation in hypertensive rats but showed no effect in normotensive rats (24). Additionally, Yamawaki et al. (25) showed that nesfatin-1 decreased nitroprusside-induced relaxation in the VEC-denuded mesenteric arteries of rats ex vivo, whereas Barutcigil et al. (23) reported opposite findings, with nesfatin-1 increasing nitroprussideinduced relaxation in the VEC-intact aortas of rats ex vivo. Collectively, these studies indicate that under VEC-intact conditions, nesfatin-1 is likely to promote vascular protection; however, under conditions in which VECs are impaired such as severe hypertension, nesfatin-1 conversely facilitates vascular injury.

Although the receptors for nesfatin-1 have not been completely identified, nesfatin-1 reportedly induces multiple molecular changes in various tissues and cells (2). However, the effects of nesfatin-1 on VECs have been largely unknown. We found that nesfatin-1 increased the phosphorylation of AMPK but not of Akt or p42/44 in cultured HUVECs. Activation of both AMPK and Akt

This work is licensed under a Creative Commons Attribution-NonCommercial 4.0 International License. ded from Bioscientifica.com at $04 / 26 / 2023$ 12:36:47PM 
A

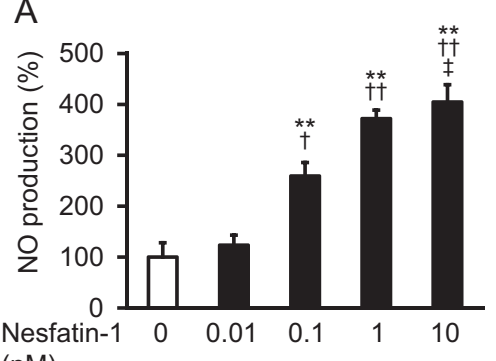

$\mathrm{B}$

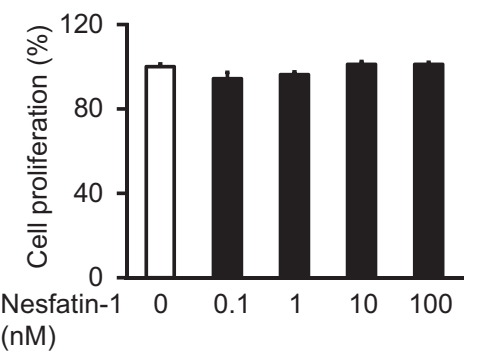

E
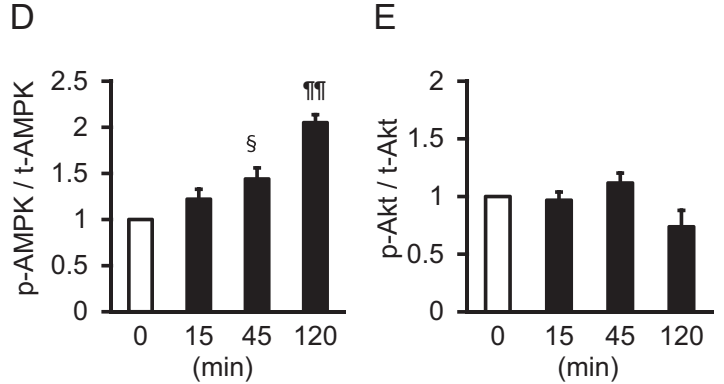

$\mathrm{H}$

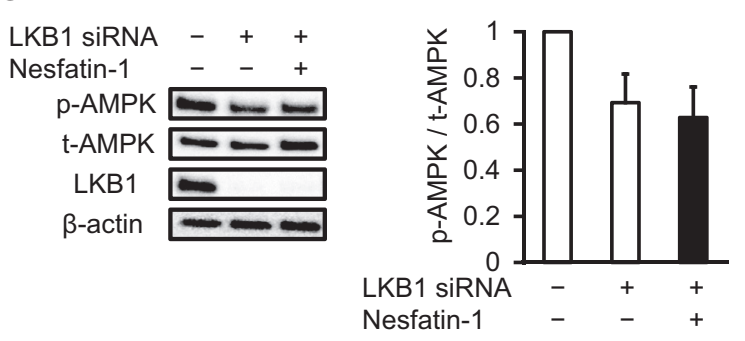

\section{Figure 3}

Nesfatin-1 increases nitric oxide production and AMPK phosphorylation in HUVECs. (A and B) Effects of nesfatin-1 on NO production (A) and cell proliferation (B). HUVECs were stimulated with vehicle or nesfatin-1 at 0.01 to $10 \mathrm{nmol} / \mathrm{L}$ for $2 \mathrm{~h}$ (A) or at 0.1 to $100 \mathrm{nmol} / \mathrm{L}$ for $24 \mathrm{~h}$ (B). (C, D, E and F) Effects of nesfatin-1 on phosphorylation of AMPK (D), Akt (E) and p42/44 (F). (G and H) Effects of LKB1 knockdown on nesfatin-1-induced phosphorylation of AMPK. HUVECs were stimulated with vehicle or nesfatin-1 at $100 \mathrm{nmol} / \mathrm{L}$ for 15,45 , or $120 \mathrm{~min}$ (C, D, E and F) or $2 \mathrm{~h}$ ( $\mathrm{G}$ and $\mathrm{H})$. Representative immunoblot bands are presented in $(\mathrm{C})$ and $(\mathrm{G})$. (A and $\mathrm{B}) n=4$ per group; (D, E and F, H) $n=3-6$ per time point or group. Phosphorylated protein levels were normalized to corresponding total protein levels. ${ }^{* *} P<0.01$ vs control; ${ }^{\dagger} P<0.05$ vs nesfatin- 1 $(0.01 \mathrm{nmol} / \mathrm{L}) ;{ }^{\dagger \dagger} P<0.01$ vs nesfatin- 1 $(0.01 \mathrm{nmol} / \mathrm{L}) ;{ }^{\ddagger} P<0.05$ vs nesfatin- $1(0.1 \mathrm{nmol} / \mathrm{L}) ;$ ${ }^{5} P<0.05$ vs $0 \mathrm{~min} ;{ }^{9} \mathrm{I} P<0.01$ vs all others. contributes to an increase in NO production via the activation of endothelial nitric oxide synthase in VECs (31). Thus, our findings indicate that AMPK but not Akt may be involved in the enhancement of endothelial NO production by nesfatin-1. Additionally, siRNA-mediated knockdown of LKB1 abolished the effects of nesfatin-1 on AMPK. LKB1 is a known upstream molecule of AMPK (36). Consistent with our findings, a recent study demonstrated that the genetic ablation of $N u c b 2$ led to reductions in the migration and invasion of colon cancer cells through inhibition of the LKB1/AMPK pathway in vitro and in vivo (37). However, additional molecular

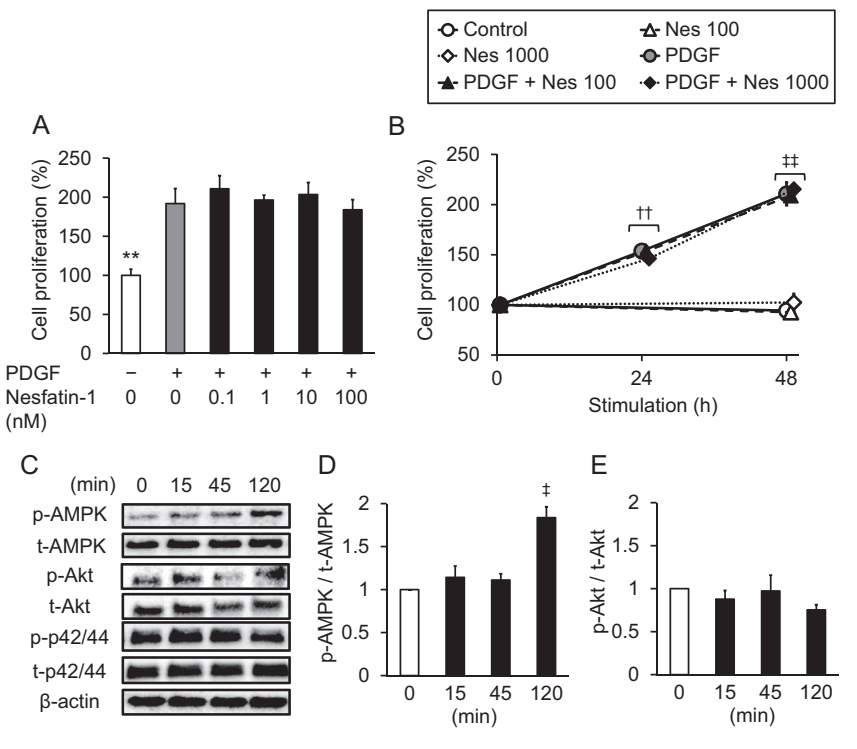

https://ec.bioscientifica.com https://doi.org/10.1530/EC-19-0120 (c) 2019 The authors Published by Bioscientifica Ltd

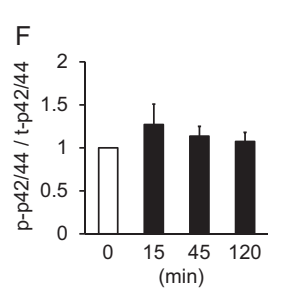

\section{Figure 4}

Nesfatin-1 increases AMPK phosphorylation but does not affect proliferation in HASMCs. (A and B) Effects of nesfatin-1 on cell proliferation. HASMCs were stimulated with vehicle or nesfatin-1 at $0.1-100 \mathrm{nmol} / \mathrm{L}$ for $48 \mathrm{~h}(\mathrm{~A})$ or at 100 or $1000 \mathrm{nmol} / \mathrm{L}$ for 24 and $48 \mathrm{~h}(\mathrm{~B})$ in the absence or presence of PDGF (20 ng/mL). (C, D, E and F) Effects of nesfatin-1 on phosphorylation of AMPK (D), Akt (E) and p42/44 (F). HASMCs were stimulated with nesfatin-1 at $100 \mathrm{nmol} / \mathrm{L}$ for 15 , 45 or $120 \mathrm{~min}$. Representative immunoblot bands are presented in (C). (A and B) $n=4$ per group; (D, $\mathrm{E}$ and F) $n=3$ per time point. Phosphorylated protein levels were normalized to corresponding total protein levels. ${ }^{* *} P<0.01$ vs all others;

${ }^{{ }^{\dagger}} P<0.01$ vs baseline; ${ }^{\ddagger} P<0.05$ vs all others; ${ }^{\ddagger \ddagger} P<0.01$ vs all others. 
mechanisms involved in the activation of LKB1 were not determined in the present study. Previous studies have reported that nesfatin-1 increases intracellular $\mathrm{Ca}^{2+}$ levels in rat hypothalamic neurons, and this effect is abolished by inhibiting cAMP-dependent protein kinase (PKA) (38). PKA has been shown to directly activate LKB1 (39); hence, PKA may potentially play a role upstream of the LKB1/AMPK pathway in nesfatin-1 receptor signaling in VECs (the proposed signaling pathway is presented in Supplementary Fig. 2).

There are several limitations of the current study. First, we evaluated the vasoprotective effects of nesfatin-1 in mouse models of wire injury. Despite widespread application in studies of peripheral arterial disease (40), the present findings cannot be directly translated to atherosclerotic CVD. However, the pathophysiology of wire-injury-induced arterial remodeling shares a number of similarities with that of atherosclerosis. Both arterial remodeling and atherosclerosis commonly exhibit intimal hyperplasia as a histological characteristic. In the process of intimal hyperplasia, the main pathophysiology is proliferation and migration of VSMCs and deposition of extracellular matrix, which result from impaired function of VECs and phenotypic switching of VSMCs $(29,41)$. Thus, our findings imply that nesfatin-1 may be a potential target for CVD and validate a further study to evaluate the cardiovascular protective effects of nesfatin-1 in animal models that are more relevant to atherosclerotic CVD. Second, we did not find antimitotic effects of nesfatin- 1 in HASMCs. Conversely, it is reported that, in the absence of growth factors, nesfatin-1 increases the migration and proliferation of human and rat aortic VSMCs via downregulation of peroxisome proliferator-activated receptor $\gamma(18,24)$. To obtain a better understanding of the effects of nesfatin-1 on VECs and VSMCs, further studies are required to clarify the signaling pathways of nesfatin- 1 receptors. Third, we did not observe obvious anti-obesity effects of nesfatin-1 in the present study, possibly because of the use of nonobese models or nesfatin-1 administration at lower doses than those reporting anorexic and vasopressor effects of nesfatin-1 $(16,17,30)$.

We demonstrated that nesfatin-1 treatment at appropriate doses suppresses peripheral artery remodeling without elevating blood pressure in mice, possibly through the enhancement of NO production and LKB1mediated activation of AMPK in VECs. Our findings indicate that nesfatin-1 may be a therapeutic target for peripheral artery disease.

\section{Supplementary data}

This is linked to the online version of the paper at https://doi.org/10.1530/ EC-19-0120.

\section{Declaration of interest}

$\mathrm{T} \mathrm{H}$ received lecture fees from AstraZeneca, Daiichi Sankyo, Eli Lilly Japan, Kowa, Mitsubishi Tanabe Pharma, MSD, Novartis Pharma, Novo Nordisk Pharma, Ono Pharmaceutical and Takeda. However, the sponsors of the lecture fees played no role in the design of the study, collection, analysis or interpretation of data or writing of the manuscript. They also did not have access to data stemming from this study. The other authors declare that there is no conflict of interest that could be perceived as prejudicing the impartiality of the research reported.

\section{Funding}

This study was supported by JSPS KAKENHI (Grant Number 16K21370).

\section{Author contribution statement}

Y M designed the study; researched and analyzed the data and drafted revised and finalized the manuscript. H S generated NUCB2-Tg mice and contributed to revision of the manuscript. $\mathrm{H} \mathrm{K}, \mathrm{T} \mathrm{S}, \mathrm{M} \mathrm{H}, \mathrm{M} \mathrm{T}$ and $\mathrm{M} \mathrm{K}$ researched and analyzed the data and contributed to revision of the manuscript. $\mathrm{H} \mathrm{O}$ and $\mathrm{T} \mathrm{H}$ contributed to the data interpretation and revision of the manuscript. All authors approved the final version of the manuscript. Y M is the guarantor of this work and is responsible for its integrity.

\section{References}

1 Oh S, Shimizu H, Satoh T, Okada S, Adachi S, Inoue K, Eguchi H, Yamamoto M, Imaki T, Hashimoto K, et al. Identification of nesfatin-1 as a satiety molecule in the hypothalamus. Nature 2006 443 709-712. (https://doi.org/10.1038/nature05162)

2 Schalla MA \& Stengel A. Current understanding of the role of nesfatin-1. Journal of the Endocrine Society 20182 1188-1206. (https:// doi.org/10.1210/js.2018-00246)

3 Yosten GL \& Samson WK. Nesfatin-1 exerts cardiovascular actions in brain: possible interaction with the central melanocortin system. American Journal of Physiology. Regulatory, Integrative and Comparative Physiology 2009297 R330-R336. (https://doi.org/10.1152/ ajpregu.90867.2008)

4 Ding S, Qu W, Dang S, Xie X, Xu J, Wang Y, Jing A, Zhang C \& Wang J. Serum nesfatin-1 is reduced in type 2 diabetes mellitus patients with peripheral arterial disease. Medical Science Monitor 2015 21 987-991. (https://doi.org/10.12659/MSM.892611)

5 Duan XL, Li L \& Li YN. Association of serum nesfatin-1 concentrations with atrial fibrillation. Kaohsiung Journal of Medical Sciences 201834 348-351. (https://doi.org/10.1016/j. kjms.2017.12.002)

6 Kuyumcu A, Kuyumcu MS, Özbay MB \& Özeke Ö. The relationship between nesfatin-1 and carotid artery stenosis. Scandinavian Cardiovascular Journal 20192 1-18. (https://doi.org/10.1080/1401743 1.2018.1547840)

7 Shen P, Han Y, Cai B \& Wang Y. Decreased levels of serum nesfatin-1 in patients with obstructive sleep apnea syndrome. Sleep and Breathing 201519 515-522. (https://doi.org/10.1007/s11325-0141039-0)

8 Zhai T, Li SZ, Fan XT, Tian Z, Lu XQ \& Dong J. Circulating nesfatin-1 levels and type 2 diabetes: a systematic review and meta-analysis.

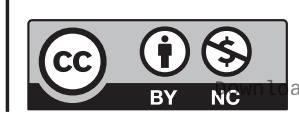

This work is licensed under a Creative Commons Attribution-NonCommercial 4.0 International License. Atribution-NonCommercial 4.0 International License. 
Journal of Diabetes Research 20172017 7687098. (https://doi. org/10.1155/2017/7687098)

9 Jiang G, Wang M, Wang L, Chen H, Chen Z, Guo J, Weng X \& Liu X. The protective effect of nesfatin-1 against renal ischemia-reperfusion injury in rats. Renal Failure 201537 882-889. (https://doi.org/10.310 9/0886022X.2015.1015426)

10 Kolgazi M, Cantali-Ozturk C, Deniz R, Ozdemir-Kumral ZN, Yuksel M, Sirvanci S \& Yeğen BC. Nesfatin-1 alleviates gastric damage via direct antioxidant mechanisms. Journal of Surgical Research 2015 193 111-118. (https://doi.org/10.1016/j.jss.2014.06.057)

11 Özsavcí D, Erşahin M, Şener A, Özakpinar ÖB, Toklu HZ, Akakín D, Şener G \& Yeğen BÇ. The novel function of nesfatin-1 as an antiinflammatory and antiapoptotic peptide in subarachnoid hemorrhageinduced oxidative brain damage in rats. Neurosurgery 201168 1699-708; discussion 1708. (https://doi.org/10.1227/NEU.0b013e318210f258)

12 Ravussin A, Youm YH, Sander J, Ryu S, Nguyen K, Varela L, Shulman GI, Sidorov S, Horvath TL, Schultze JL, et al. Loss of nucleobindin-2 causes insulin resistance in obesity without impacting satiety or adiposity. Cell Reports 201824 1085-1092.e6. (https://doi.org/10.1016/j.celrep.2018.06.112)

13 Solmaz A, Bahadır E, Gülçiçek OB, Yiğitbaş H, Çelik A, Karagöz A, Özsavcı D, Şirvancı S \& Yeğen BÇ. Nesfatin-1 improves oxidative skin injury in normoglycemic or hyperglycemic rats. Peptides $2016 \mathbf{7 8}$ 1-10. (https://doi.org/10.1016/j.peptides.2015.12.006)

14 Tang $\mathrm{CH}, \mathrm{Fu} \mathrm{XJ}, \mathrm{Xu} \mathrm{XL}$, Wei XJ \& Pan HS. The anti-inflammatory and anti-apoptotic effects of nesfatin-1 in the traumatic rat brain. Peptides 201236 39-45. (https://doi.org/10.1016/j. peptides.2012.04.014)

15 Tasatargil A, Kuscu N, Dalaklioglu S, Adiguzel D, Celik-Ozenci C, Ozdem S, Barutcigil A \& Ozdem S. Cardioprotective effect of nesfatin-1 against isoproterenol-induced myocardial infarction in rats: role of the Akt/GSK-3 $\beta$ pathway. Peptides 201795 1-9. (https:// doi.org/10.1016/j.peptides.2017.07.003)

16 Ayada C, Turgut G, Turgut S \& Güçlü Z. The effect of chronic peripheral nesfatin-1 application on blood pressure in normal and chronic restraint stressed rats: related with circulating level of blood pressure regulators. General Physiology and Biophysics 2015 34 81-88. (https://doi.org/10.4149/gpb_2014032)

17 Osaki A \& Shimizu H. Peripheral administration of nesfatin-1 increases blood pressure in mice. Hypertension Research 201437 185-186. (https://doi.org/10.1038/hr.2013.122)

18 Zhang JR, Lu QB, Feng WB, Wang HP, Tang ZH, Cheng H, Du Q, Wang YB, Li KX \& Sun HJ. Nesfatin-1 promotes VSMC migration and neointimal hyperplasia by upregulating matrix metalloproteinases and downregulating PPAR $\gamma$. Biomedicine and Pharmacotherapy 2018 102 711-717. (https://doi.org/10.1016/j.biopha.2018.03.120)

19 National Research Council Committee for the Update of the Guide for the Care and Use of Laboratory Animals. Guide for the Care and Use of Laboratory Animals, 8th ed. Eds CH Fletcher \& R Crossgrove. New York, NY, USA: National Academies Press, 2011.

20 Shimizu H, Tanaka M \& Osaki A. Transgenic mice overexpressing nesfatin/nucleobindin-2 are susceptible to high-fat diet-induced obesity. Nutrition and Diabetes 20166 e201. (https://doi.org/10.1038/ nutd.2015.42)

21 Sata M, Maejima Y, Adachi F, Fukino K, Saiura A, Sugiura S, Aoyagi T, Imai $\mathrm{Y}$, Kurihara $\mathrm{H}$, Kimura $\mathrm{K}$, et al. A mouse model of vascular injury that induces rapid onset of medial cell apoptosis followed by reproducible neointimal hyperplasia. Journal of Molecular and Cellular Cardiology 200032 2097-2104. (https://doi.org/10.1006/ jmcc.2000.1238)

22 Kushima H, Mori Y, Koshibu M, Hiromura M, Kohashi K, Terasaki M, Fukui T \& Hirano T. The role of endothelial nitric oxide in the anti-restenotic effects of liraglutide in a mouse model of restenosis Cardiovascular Diabetology 201716 122. (https://doi.org/10.1186/ s12933-017-0603-x)
23 Barutcigil A \& Tasatargil A. Effects of nesfatin-1 on atrial contractility and thoracic aorta reactivity in male rats. Clinical and Experimental Hypertension 201840 414-420. (https://doi.org/10.1080/10641963.20 17.1384487)

24 Lu QB, Wang HP, Tang ZH, Cheng H, Du Q, Wang YB, Feng WB, Li KX, Cai WW, Qiu LY, et al. Nesfatin-1 functions as a switch for phenotype transformation and proliferation of VSMCs in hypertensive vascular remodeling. Biochimica et Biophysica Acta. Molecular Basis of Disease 20181864 2154-2168. (https://doi. org/10.1016/j.bbadis.2018.04.002)

25 Yamawaki H, Takahashi M, Mukohda M, Morita T, Okada M \& Hara Y. A novel adipocytokine, nesfatin-1 modulates peripheral arterial contractility and blood pressure in rats. Biochemical and Biophysical Research Communications 2012418 676-681. (https://doi. org/10.1016/j.bbrc.2012.01.076)

26 Nagashima M, Watanabe T, Terasaki M, Tomoyasu M, Nohtomi K, Kim-Kaneyama J, Miyazaki A \& Hirano T. Native incretins prevent the development of atherosclerotic lesions in apolipoprotein $\mathrm{E}$ knockout mice. Diabetologia 201154 2649-2659. (https://doi. org/10.1007/s00125-011-2241-2)

27 Mori Y, Kushima H, Koshibu M, Saito T, Hiromura M, Kohashi K, Terasaki M, Seino Y, Yamada Y \& Hirano T. Glucose-dependent insulinotropic polypeptide suppresses peripheral arterial remodeling in male mice. Endocrinology 2018159 2717-2732. (https://doi. org/10.1210/en.2018-00336)

28 Mori Y, Shimizu H, Kushima H, Hiromura M, Terasaki M, Tanaka M, Osaki A \& Hirano T. Increased blood pressure in nesfatin/ nuclebindin-2-transgenic mice. Hypertension Research $2017 \mathbf{4 0}$ 861-867. (https://doi.org/10.1038/hr.2017.61)

29 Otsuka F, Finn AV, Yazdani SK, Nakano M, Kolodgie FD \& Virmani R. The importance of the endothelium in atherothrombosis and coronary stenting. Nature Reviews. Cardiology 20129 439-453. (https://doi.org/10.1038/nrcardio.2012.64)

30 Shimizu H, Oh S, Hashimoto K, Nakata M, Yamamoto S, Yoshida N, Eguchi H, Kato I, Inoue K, Satoh T, et al. Peripheral administration of nesfatin-1 reduces food intake in mice: the leptin-independent mechanism. Endocrinology 2009150 662-671. (https://doi. org/10.1210/en.2008-0598)

31 Garcia V \& Sessa WC. Endothelial NOS: perspective and recent developments. British Journal of Pharmacology 2019176 189-196. (https://doi.org/10.1111/bph.14522)

32 Cooney R, Hynes SO, Sharif F, Howard L \& O’Brien T. Effect of gene delivery of NOS isoforms on intimal hyperplasia and endothelial regeneration after balloon injury. Gene Therapy 200714 396-404. (https://doi.org/10.1038/sj.gt.3302882)

33 Tanner FC, Meier P, Greutert H, Champion C, Nabel EG \& Lüscher TF. Nitric oxide modulates expression of cell cycle regulatory proteins: a cytostatic strategy for inhibition of human vascular smooth muscle cell proliferation. Circulation 2000101 1982-1989. (https://doi.org/10.1161/01.CIR.101.16.1982)

34 Tsihlis ND, Oustwani CS, Vavra AK, Jiang Q, Keefer LK \& Kibbe MR. Nitric oxide inhibits vascular smooth muscle cell proliferation and neointimal hyperplasia by increasing the ubiquitination and degradation of UbcH10. Cell Biochemistry and Biophysics 201160 89-97. (https://doi.org/10.1007/s12013-011-9179-3)

35 Kumar A \& Lindner V. Remodeling with neointima formation in the mouse carotid artery after cessation of blood flow. Arteriosclerosis, Thrombosis, and Vascular Biology 199717 2238-2244. (https://doi. org/10.1161/01.ATV.17.10.2238)

36 Carling D, Sanders MJ \& Woods A. The regulation of AMP-activated protein kinase by upstream kinases. International Journal of Obesity 200832 S55-S59. (https://doi.org/10.1038/ijo.2008.124)

37 Kan JY, Yen MC, Wang JY, Wu DC, Chiu YJ, Ho YW \& Kuo PL. Nesfatin-1/nucleobindin-2 enhances cell migration, invasion, and epithelial-mesenchymal transition via LKB1/AMPK/TORC1/ZEB1 https://ec.bioscientifica.com https://doi.org/10.1530/EC-19-0120 (c) 2019 The authors Published by Bioscientifica Ltd

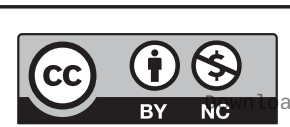

This work is licensed under a Creative Commons Attribution-NonCommercial 4.0 International License. ded from Bioscientifica com at 04/26/2023 12:36:47PM 
pathways in colon cancer. Oncotarget 20167 31336-31349. (https:// doi.org/10.18632/oncotarget.9140)

38 Brailoiu GC, Dun SL, Brailoiu E, Inan S, Yang J, Chang JK \& Dun NJ. Nesfatin-1: distribution and interaction with a $G$ protein-coupled receptor in the rat brain. Endocrinology 2007148 5088-5094. (https:// doi.org/10.1210/en.2007-0701)

39 Collins SP, Reoma JL, Gamm DM \& Uhler MD. LKB1, a novel serine/threonine protein kinase and potential tumour suppressor, is phosphorylated by cAMP-dependent protein kinase (PKA) and prenylated in vivo. Biochemical Journal 2000345 673-680. (https:// doi.org/10.1042/bj3450673)

40 Touchard AG \& Schwartz RS. Preclinical restenosis models: challenges and successes. Toxicologic Pathology 200634 11-18. (https://doi.org/10.1080/01926230500499407)

41 Rzucidlo EM, Martin KA \& Powell RJ. Regulation of vascular smooth muscle cell differentiation. Journal of Vascular Surgery 200745 (Supplement A) A25-A32. (https://doi.org/10.1016/j. jvs.2007.03.001)

Received in final form 11 March 2019

Accepted 1 April 2019

Accepted Preprint published online 2 April 2019 